\title{
The origin of human chromosome 2 analyzed by comparative chromosome mapping with a DNA microlibrary
}

\author{
J. Wienberg, A. Jauch, H.-J. Lüdecke, G. Senger, \\ B. Horsthemke, U. Claussen, T. Cremer, N. Arnold \\ \& C. Lengauer
}

Received 7 March 1994; received in revised form 5 May 1994; Accepted for publication by M. Schmid 5 May 1994

\begin{abstract}
Fluorescence in situ hybridization (FISH) of microlibraries established from distinct chromosome subregions can test the evolutionary conservation of chromosome bands as well as chromosomal rearrangements that occurred during primate evolution and will help to clarify phylogenetic relationships. We used a DNA library established by microdissection and microcloning from the entire long arm of human chromosome 2 for fluorescence in situ hybridization and comparative mapping of the chromosomes of human, great apes (Pan troglodytes, Pan paniscus, Gorilla gorilla, Pongo pygmaeus) and Old Worid monkeys (Macaca fuscata and Cercopithecus aethiops). Inversions were found in the pericentric region of the primate chromosome $2 p$ homologs in great apes, and the hybridization pattern demonstrates the known phylogenetically derived telomere fusion in the line that leads to human chromosome 2. The hybridization of the $2 q$ microlibrary to chromosomes of Old World monkeys gave a different pattern from that in the gorilla and the orang-utan, but a pattern similar to that of chimpanzees. This suggests convergence of chromosomal rearrangements in different phylogenetic lines.
\end{abstract}

Key words: evolution, fluorescent in situ hybridization, microdissection, phylogeny, primates

\section{Introduction}

Comparative banding analysis between human chromosomes and the karyotype of great apes suggests that human chromosome 2 was derived by a fusion of two primate homologs (Dutrillaux 1979, Yunis \& Prakash 1982). This has been confirmed by fluorescence in situ hybridization (FISH) of a human chromosome 2-specific DNA library to primate chromosomes (Wienberg et al. 1990, 1992, Jauch et al. 1992). However, the exact phylogenetic interpretation of the chromosome rearrangements noted between these species is still not clear. Owing to the presence of pericentromeric heterochromatin in great ape chromosomes, it is difficult to match the homologous bands close to the centromeres by classical banding studies. From high-resolution chromosome banding, Yunis \& Prakash (1982) proposed a common derived (synapomorphic) pericentric inversion in the homolog to human chromosome $2 \mathrm{p}$ in a suggested chimpanzee/human phylogenetic lineage, which was followed by a fusion event in the human karyotype.

A more secure demonstration of the homology between individual bands is provided by fluorescence in situ hybridization of chromosome band-specific DNA libraries (Lengauer et al. 1991a, b; Trautmann et al. 1991). The construction of such libraries is possible by microdissection and microcloning (Lüdecke et al. 1989). We have used a microlibrary established from the long arm of human chromosome 2 for comparative chromosome mapping of human and primate species. The chromosomes of Old World monkeys were used as an 'outgroup' to analyze the direction of chromosome changes that occurred during hominoid evolution.

J. Wienberg (corresponding author) and N. Arnold are at the Institut für Anthropologie und Humangenetik, Universität München, Richard-Wagner Strasse 10/1, D-80333 Munich, Germany. Fax: $(+49) 895203$ 286. A. Jauch is at the Institut für Humangenetik und Anthropologie, Universität Heidelberg, Im Neuenheimer Feld 328, D-69120 Heidelberg, Germany. H.-J. Lüdecke and B. Horsthemke are at the Institut für Humangenetik, Universitätsklinikum Essen, Hufelandstr. 55, D-45122 Essen, Germany. G. Senger and U. Claussen are at the Institut für Humangenetik und Anthropologie, Universität Jena, Kollegiengasse 10, D-07740 Jena, Germany. C. Lengauer is at the Institut für Humangenetik und Anthropologie, Universität Heidelberg, Im Neuenheimer Feld 328, D-69120, Germany, and is currently at the Research Institute of Molecular Pathology, Dr. Bohr Gasse 7, A-1030 Vienna, Austria. 


\section{$J$. Wienberg et al.}

\section{Material and methods}

\section{Chromosome preparations}

The present study includes chromosome preparation from human (HSA), Pan troglodytes (PTR), P. paniscus (PPA), Gorilla gorilla (GGO), Pongo pygmaeus (PPY), Macaca fuscata (MFU) and Cercopithecus aethiops (CAE). Metaphase chromosomes were made from standard preparations of phytohemagglutinin (PHA)-stimulated peripheral lymphocytes or from lymphoblastoid cell lines established from blood samples kindly provided by the Munich Zoo 'Tiergarten Hellabrunn' or from Drs W. Schempp, Freiburg, Germany, M. Schmid, Würzburg, Germany, and R. Stanyon, Genoa, Italy. Microscopic slides were prepared by standard techniques. Before using the lymphoblastoid cell lines in this study, chromosomes were checked for aberrations and were found to be normal diploid, except for one of the gorilla cell lines, which showed a translocation in the chromosome homologous to human chromosome $2 \mathrm{q}$.

\section{DNA probe}

The microlibrary was established from the entire long arm of the human chromosome 2 . Microdissection was performed essentially as described by Lüdecke et al. (1989) except that the chromosomes were not GTG banded. Twenty chromosome fragments were pooled into the collection drop and microreactions were performed as previously described (Lüdecke et al. 1989). An aliquot (100 ng) of the primary amplification products was biotin labeled in a $100-\mu \mathrm{l}$ assay containing $1 \mu \mathrm{M}$ universal $\mathrm{M} 13 / \mathrm{pUC}$ sequencing and reverse sequencing primer, $10 \mathrm{mM}$ Tris-HCL ( $\mathrm{pH}$ 8.4), $50 \mathrm{mM} \mathrm{KCl}, 1.5 \mu \mathrm{M} \mathrm{MgCl}, 0.001 \%$ gelatin, $200 \mu \mathrm{M}$ dATP, dGTP and dCTP, $100 \mu \mathrm{M}$ dTTP, $150 \mu \mathrm{M}$ bio-11-dUTP and 2.5 units of Thermus aquaticus DNA polymerase. After initial denaturation at $96^{\circ} \mathrm{C}$ for $3 \mathrm{~min}, 25$ cycles of polymerase chain reaction (PCR) were carried out with denaturation at $96^{\circ} \mathrm{C}$ for $1 \mathrm{~min}$, annealing at $47^{\circ} \mathrm{C}$ for $1 \mathrm{~min}$ and extension at $72^{\circ} \mathrm{C}$ for $2 \mathrm{~min}, 10 \mathrm{~s}$.

\section{Chromosome banding}

Chromosome banding prior to FISH was achieved according to Klever et al. (1991). Briefly, after routine GTG banding, metaphases were photographed (Agfa-Ortho 25), destained with fixative (methanol-acetic acid 3:1) and post-fixed with $4 \%$ formaldehyde in phosphate-buffered saline (PBS) for $15 \mathrm{~min}$. Post-fixation was essential to preserve chromosome morphology in subsequent FISH experiments.

\section{Fluorescence in situ hybridization}

About $200 \mathrm{ng}$ of PCR amplified biotin-labeled probe was co-precipitated with $10 \mu \mathrm{g}$ of salmon sperm DNA and $30 \mu \mathrm{g}$ of Cot-1 DNA and the pellet was redissolved in 50\% formamide, $1 \times \mathrm{SSC}$ and $10 \%$ dextran sulfate. The biotin-labeled probe was hybridized under suppression conditions (Cremer et al. 1988, Lichter et al. 1988, Pinkel et al. 1988) and detected with fluorescein-conjugated avidin. Analysis was performed using a Zeiss Axiophot microscope equipped with epifluorescence and the filter combination for fluorescein, rhodamine and DAPI (4',6-diaminidino-2phenylindole), Chromosomes were photographed with Agfachrome (1000 ASA) color slide films or Kodak T-MAX black and white (400 ASA) films.

The photographed hybridization signals were superimposed over the previous photographed Gbanded chromosomes to identify the marked chromosomes and the position of signals along the chromosomes.

\section{Results}

Fluorescence in situ hybridization (FISH) of the established $2 q$ microlibrary to human lymphocyte metaphase spreads resulted as expected in a specific delineation of the long arm of human chromosome 2 (Figure 1a). In metaphase spreads from great apes, gibbons and Old World monkeys different hybridization patterns were observed.

In both chimpanzees ( $P$. troglodytes and $P$. paniscus), the entire chromosomes PTR 13 and PPA 13 were stained, except for a small part of the short arm that remained unlabeled. On the chromosomes PTR 12 and PPA 12 hybridization signals were restricted to the short arm also, leaving a small unlabeled band (Figure 1c), which may represent telomeric heterochromatin.

The hybridization pattern of the gorilla chromosome GGO 11 was similar to that found on the chimpanzee chromosomes PTR 13 and PPA 13 except that the unlabeled telomeric band was more pronounced. The labeling of the gorilla chromosome GGO 12 was different from that of both chimpanzee chromosomes PTR 12 and PPA 12, which are supposed to be homologs. Hybridization signals were found on the long arm of gorilla chromosome GGO 12 close to the centromere, whereas the short arm was not labeled (Figure 1e). A bright band with the counterstain DAPI indicates that the short arm of GGO 12 may be totally heterochromatic (not shown). The two orang-utan homologs (PPY 12 and PPY 11) were labeled as in the gorilla, except for the telomeric heterochromatin, which is not present in this species (Figure 1g).

Hybridization of the $2 q$ microlibrary to Old World monkey chromosomes gave a similar pattern as in the two chimpanzees. In both species analyzed one submetacentric chromosome pair (MFU15, CAE16) 
was entirely labeled including the short arms (Figure 1h and i). On another submetacentric chromosome pair (MFU9, CAE13) labeling was restricted to the short arm, again except for the heterochromatic region in CAE. A schematic summary of the hybridization patterns found in hominoid and Old World monkey chromosomes is shown in Figure 2.

\section{Discussion}

Recently we have shown that FISH of DNA microlibraries established from microdissected human chromosome regions can be used as a tool to confirm the origin of the bulk DNA sequences from the corresponding region (Lengauer et al. 1991a, b, Trautmann et al. 1991). This present paper demonstrates the specific visualization of human chromosome $2 q$ material on homologous primate chromosomes using a microlibrary established after microdissection of the entire long arm of human chromosome 2. The hybridization pattern allows a direct evaluation of chromosome rearrangements that occurred during hominoid evolution.

In all great ape and Old World monkey species analyzed, the human $2 q$ microlibrary 'paints' one chromosome pair entirely. By conventional banding patterns, these chromosomes were already supposed to be homologous to human chromosome $2 \mathrm{q}$. In addition to this painting signal, a small segment of another great ape and Old World monkey chromosome pair was labeled. Conventional banding pattern analysis had assigned this chromosome pair to be homologous to human chromosome $2 \mathrm{p}$. However, the present FISH results of the $2 \mathrm{q}$ microlibrary show that this chromosome pair also contains a chromosome segment homologous to human chromosome $2 q$. The results clearly demonstrate that the fusion point of the two ancestral chromosomes of human chromosome 2 was beyond the centromere in the long arm. From the length of the labeled segment we suggest that the fusion point was somewhere close to human band $2 q 13$.

The small labeled segment on one of the chromosomes homologous to human chromosome 2 is found in the short arm in chimpanzees and in the long arm of gorilla and orang-utan chromosomes (Figure 2). The hybridization pattern indicates that this segment was moved by one or multiple pericentric inversions. FISH of the $2 \mathrm{q}$ microlibrary on great ape chromosomes supports the simplest explanation for the origin of human chromosome 2, which would implicate a telomere-to-telomere fusion of two submetacentric chromosomes similar to that found in the chimpanzees (Dutrillaux 1979, Yunis \& Prakash 1982). Thus, the centromere of human chromosome 2 should have been derived from a chromosome similar to PTR 12 or PPA 12, while the centromere from the chromosome similar to PTR 13 or PPA 13 was inactivated or lost (Dutrillaux 1979). This hypothesis is supported by recent in situ hybridization experiments with centromere and telomere DNA sequences of human chromosome 2 (Wells et al. 1990, Ijdo et al. 1991, Avarello et al. 1992, Baldini et al. 1993). Close to the assumed fusion point (2q13) a relict of an alphoid domain was found by in situ hybridization of a satellite DNA clone under low hybridization stringency (Avarello et al. 1992, Baldini et al. 1993). This alphoid domain may indicate the presence of the inactivated centromere in human band $2 q 21$. In addition, proximal to 2q21, telomeric sequences were found in band $2 q 13$ by in situ hybridization of DNA probes containing inverted arrays of the vertebrate telomeric repeat in a head-to-head arrangement (Wells et al. 1990, Ijdo et al. 1991). This band should therefore represent the fusion point of the telomeres of the two primate homologs.

The FISH patterns of the $2 \mathrm{q}$ microlibrary indicate that pericentric inversion(s) altered the morphology of great ape chromosomes, homologous to human chromosome $2 \mathrm{p}$. To show the direction of chromosome changes during human and great ape evolution we also analyzed the FISH patterns for the $2 q$ microlibrary in Old World monkeys as an 'outgroup'. In particular, the hybridization pattern of the small segment in respect to the centromere in the 'outgroup' would allow the origin of the assumed pericentric inversion to be defined.

If the FISH pattern in the 'outgroup' had been similar to that found in orang-utan and gorilla, then the results would have supported the assumed evolutionary-derived (synapomorphic) inversion in the chromosome 2p homologs (Dutrillaux 1979, Yunis \& Prakash 1982). This would have phylogenetically linked human and chimpanzees after their divergence from the gorilla line.

However, the hybridization patterns of the $2 q$ microlibrary found on the chromosomes of Old World monkeys (M. fuscata, C. aethiops) were similar to that found in the chimpanzees. The signal of the small band on the chromosome $2 p$ homolog was found in the corresponding short arms. Therefore, the FISH patterns in the 'outgroup' indicate that the chromosome form found in the chimpanzees should be considered as the ancestral form, while the chromosome $2 \mathrm{p}$ homologs in gorilla and orang-utan experienced convergent chromosomal mutations for this inversion.

Convergent chromosomal rearrangements may be more common than usually assumed (Stanyon et al. 1994). Similar results could be obtained recently by comparative mapping of DNA probes derived from the V-kappa immunoglobulin genes, which map on both human chromosome $2 p$ and $2 q$ close to the centromere (Arnold et al. submitted). Therefore the phylogeny of human chromosome 2 and its homologs in the great apes cannot be used to discriminate between the recent most favored phylogenetic trees linking either human and chimpanzee or chimpanzee and gorilla. 


\section{$J$. Wienberg et al.}
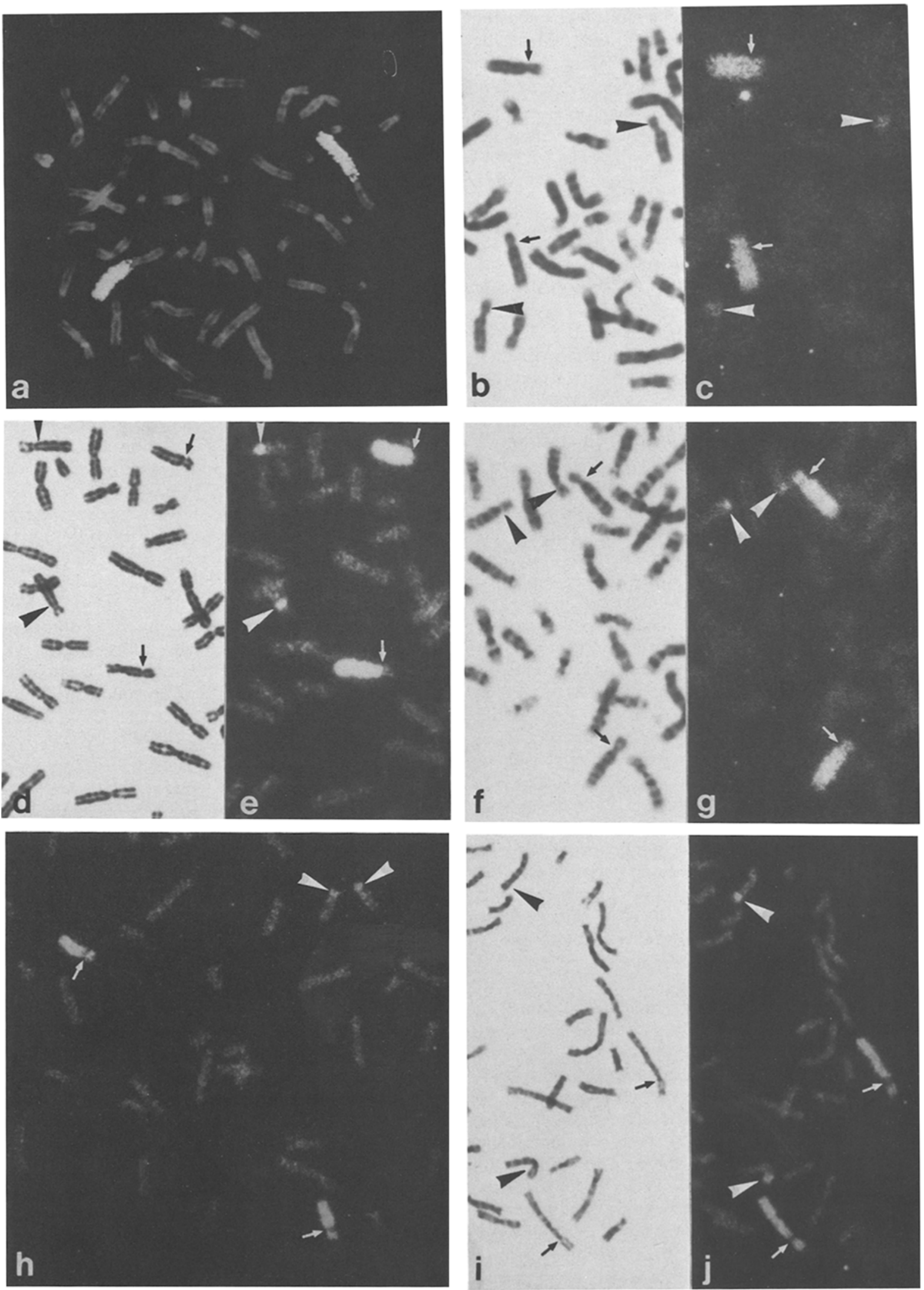
An alternative hypothesis considers the possibility that ancestral populations could have been polymorphic for these chromosome variants. Different chromosome variants may have become fixed in different phylogenetic lines (Stanyon \& Chiarelli 1983). Yet, random fixation of polymorphic chromosome forms in the different phylogenetic lines would not allow a parsimonious interpretation. This hypothesis obtains support from chromosome polymorphisms still found in human populations. In about $0.1 \%$ of humans a pericentric inversion is found on chromosome 2 involving the segment $2 \mathrm{p} 11-2 \mathrm{q} 13$, which is close to the assumed breakpoints of the pericentric inversion assumed in primate homologs (for review see Djalali et al. 1986). In Sephardic Jews this inversion has a frequency of $0.8 \%$ and even homozygotic carriers with-

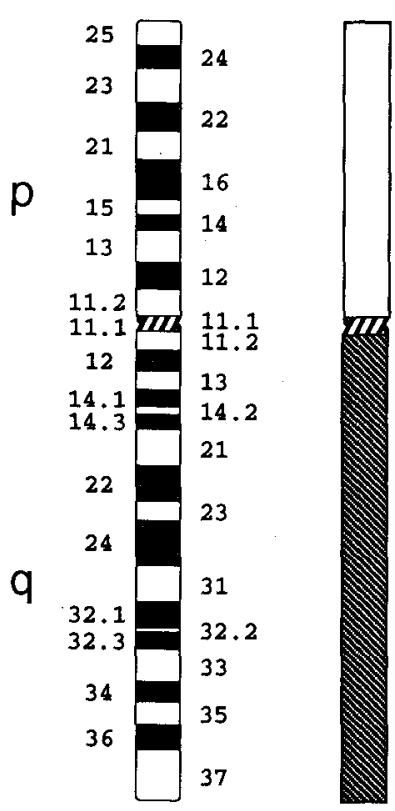

HSA 2

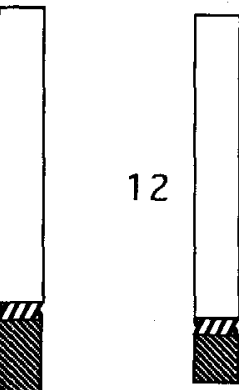

13
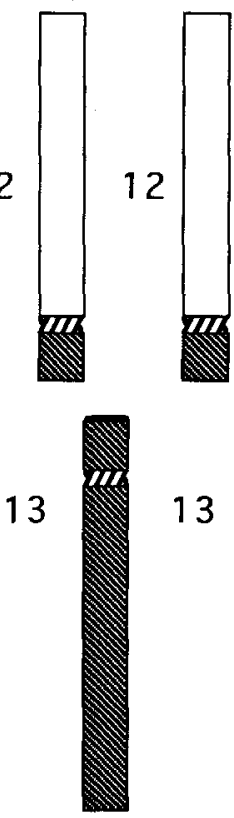

13

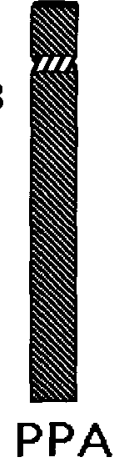

12

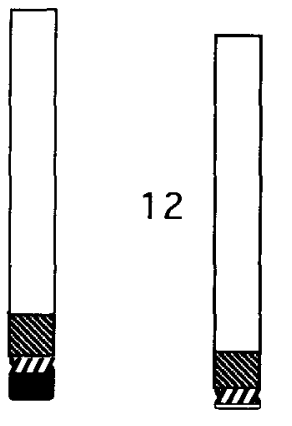

11



GGO

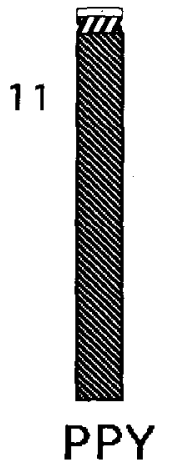

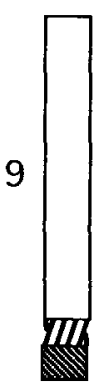
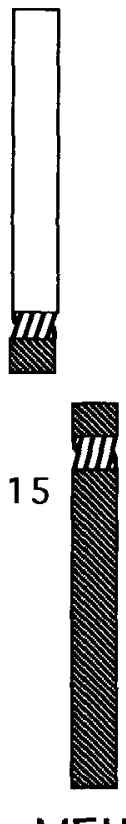

16
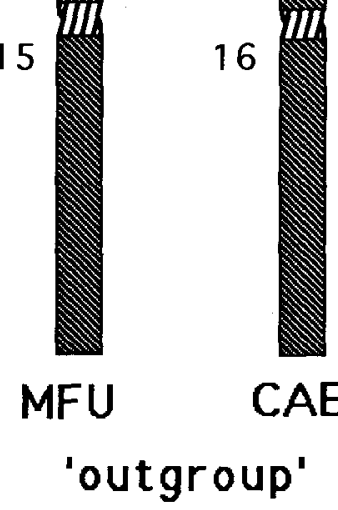

Figure 2. Schematic summary of the hybridization patterns after FISH with the human $2 q$ microlibrary to great ape and Old World monkey chromosomes. The human chromosome 2 idiogram (left) and the schematic drawing of the hybridization patterns are shown for human (HSA) and primate homologs [Pan troglodytes (PTR), P. paniscus (PPA), Gorilla gorilla (GGO), Pongo pygmaeus (PPY), Macaca fuscata (MFU) and Cercopithecus aethiops (CAE)], without indicating the chromosome banding. The numbers beside the ape chromosomes are the chromosomal nomenclature within these species. The painted chromosome regions are indicated by a hatched pattern. Telomeric C-banding in PTR, PPA, GGO and CAE is indicated by black bands. Note that the hybridization pattern on the 'classical human chromosome $2 p$ homologs' in both chimpanzee species is more similar to the pattern found in the 'outgroup' (MFU and CAE) than that in the gorilla or in the orang-utan. In these species the signals are found in the corresponding long arm.

Figure 1. FISH with a $2 q$ microlibrary to human and primate chromosomes $(\mathbf{a}-\mathbf{j})$. a Painting of the long arm of human chromosome 2 shows the origin of the DNA microclone library. b \& $\mathbf{c}$ Hybridization to chromosomes of Pan troglodytes (c). Chromosome pair 13 is painted entirely (arrows), demonstrating the homology to human chromosome $2 q$ as identified from its G-banding pattern (b). In addition, a small segment (arrowheads) of chromosome pair 12 is also painted. Note that the labeled segment spans the short arm of the chimpanzee chromosome. The position of the centromere was identified by $\mathrm{G}$ banding (b) and DAPI staining (not shown). d \& e Painted chromosomes of Gorilla gorilla (e). Chromosome pair 11 is painted entirely (arrows), whereas a small labeled band can be detected in the long arm of the gorilla chromosome 12 (arrowheads). Again, the centromere was identified by G-banding (d) and DAPI staining (not shown). $\mathbf{f} \& \mathbf{g}$ Painted chromosomes of Pongo pygmaeus (g). Chromosome pair 11 is painted entirely (arrows), whereas a small labeled band can be detected in the long arm of the pongo chromosome 12 (arrowheads). The position of the centromere was identified by G-banding ( $\mathbf{f}$ ) and DAPI staining (not shown). h-j Hybridization to chromosomes of Old World monkeys. Chromosomes of Macaca fuscata (h) and Cercopithecus aethiops (i \& i). Both species show a similar hybridization pattern as found in Pan troglodytes. One chromosome pair (15 and 16 respectively) is entirely painted (arrows). A small segment of the short arm of chromosome 9 and 13 respectively is painted in both species indicating its human chromosome $2 q$ homology (arrowheads). The centromeres were identified by G-banding (i) and DAPI staining (not shown). 
out any clinical abnormalities have been observed (Gelman-Kohan et al. 1993). Inversion polymorphisms in general are still found in hominoid karyotypes. The orang-utan is polymorphic for inversions between and within subspecies (de Boer \& Seuànez 1982). For example, the gibbons analyzed by in situ hybridization of chromosome-specific DNA libraries could all be distinguished on the basis of a chromosome 8 polymorphism (Jauch et al. 1992). All 44-chromosome gibbon species share this polymorphism (Stanyon et al 1987), indicating that it is not transient but has survived even speciation events.

The decision as to whether the inversions found in the different phylogenetic lines are the same or simply the result of apparent convergence due to similar breakpoints can be made on the molecular level. Such rearrangements can be defined by FISH with DNA probes that span the breakpoints (Ried et al. 1991, Lengauer et al. 1992). The molecular analysis of breakpoint regions will help to ascertain chromosomal rearrangements that occurred during primate evolution and will finally clarify phylogenetic relationships at the molecular level.

\section{Acknowledgements}

We would like to thank A. Wiegenstein for photographic work and R. Stanyon for suggestions and discussions. This work was supported by a grant from the Dutsche Forschungsgemeinschaft.

\section{References}

Arnold N, Wienberg J, Ermert K, Zachau H-G Comparative mapping of human and great ape chromosomes by in situ hybridization of DNA probes derived from the V-kappa immunoglobulin genes. Genomics, submitted.

Avarello R, Pedicini A, Caiulo A, Zuffardi O, Fraccaro M (1992) Evidence for an ancestral alphoid domain on the long arm of human chromosome 2. Hum Genet 89: 247-249.

Baldini A, Ried T, Shridhar V et al. (1993) An alphoid DNA sequence conserved in all human and great ape chromosomes: evidence for ancient centromeric sequences at human chromosomal regions $2 \mathrm{q} 21$ and $9 \mathrm{q} 13$. Hum Genet $\mathbf{9 0}$ : 577-583.

Boer de LEM, Seuánez HN (1982) The chromosomes of the orangutan and their relevance to the conservation of the spezies. In: de Boer LEM, ed. The Orangutan. Its Biology and Conservation. The Hague: Junk, pp 135-170.

Cremer T, Lichter P, Borden J, Ward DC, Manuelidis L (1988) Detection of chromosome aberrations in metaphase and interphase tumour cells by in situ hybridization using chromosome specific library probes. Hum Genet 80: 235-246.

Djalali M, Steinbach P, Bullerdiek J, Holmes-Siedle M, Verschraegen-Spae MR, Smith A (1986) The significance of pericentric inversions of chromosome 2. Hum Genet 72: 32-36.

Dutrillaux B (1979) Chromosomal evolution in primates. Tentative phylogeny from Microcebus murinus (prosimian to man. Hum Genet 48: 251-314.

Gelman-Kohan Z, Rosensaft J, Nisani B-C, Chemke J (1993) Homozygosity for inversion (2) (p12q14). Hum Genet 92: 427.
Ijdo JW, Baldini A, Ward DC, Reeders ST, Wells RA (1991) Origin of human chromosome 2: an ancestral telomere-telomere fusion. Proc Natl Acad Sci USA 88: 9051-9055.

Jauch A, Wienberg J, Stanyon R et al. (1992) Reconstruction of genomic rearrangements in great apes and gibbons by chromosome painting. Proc Natl Acad Sci USA 89: 8611-8615.

Klever M, Grond-Ginsbach C, Scherthan H, Schroeder-Kurth T (1991) Chromosomal in situ suppression hybridization after Giemsa banding. Hum Genet 86: 484-486.

Lengauer C, Lüdecke H-J, Wienberg J, Cremer T, Horsthemke B (1991a) Comparative chromosome band mapping in primates by in situ suppression hybridization of band specific DNA microlibraries. Hum Evol 6: 67-71.

Lengauer C, Eckelt A, Weith A et al. (1991b) Painting of defined chromosomal regions by in situ suppression hybridization of libraries from laser-microdissected chromosomes. Cytogenet Cell Genet 56: 27-30.

Lengauer C, Riethman HC, Speicher M et al. (1992) Metaphase and interphase cytogenetics with Alu-PCR amplified YAC clones containing the BCR-gene and the protooncogenes craf-1, c-fms, c-erbB-2. Cancer Res 52: 2590-2596.

Lichter P, Cremer T, Borden J, Manuelidis L, Ward DC (1988) Delineation of individual human chromosomes in metaphase and interphase cells by in situ suppression hybridization using recombinant DNA libraries. Hum Genet 80: 224-234.

Lüdecke H-J, Senger G, Claussen U, Horsthemke B (1989) Cloning defined regions of the human genome by microdissection of banded chromosomes and enzymatic amplification. Nature 338: 348-350.

Pinkel D, Landegent J, Collins C et al. (1988) Fluorescence in situ hybridization with human chromosome specific libraries: detection of trisomy 21 and translocation of chromosome 4. Proc Natl Acad Sci USA 85: 9138-9142.

Ried T, Lengauer C, Cremer T et al. (1991) Specific metaphase and interphase detection of the breakpoint region in 8q24 of Burkitt lymphoma cells by triple colour fluorescence in situ hybridization. Genes, Chromosomes and Cancer 4: 1-6.

Stanyon R, Chiarelli B (1983) Mode and tempo in primate chromosome evolution: implications for hylobatid phylogeny. J Hum Evol 12: 305-315.

Stanyon R, Sineo L, Chiarelli B, Camperio-Ciani A, Haimoff AR, Mootnick EH (1987) Banded karyotypes of the 44-chromosome Gibbons. Folia Primatol 48: 56-64.

Stanyon R, Arnold N, Koehler U, Bigoni F, Wienberg J (1994) Chromosomal painting shows that 'marked chromosomes' in lesser apes and Old World monkeys are not homologous and evolved by convergence. Cytogenet Cell Genet, in press.

Trautmann U, Leuteritz G, Senger G, Claussen U, Ballhausen WG (1991) Detection of APC region-specific signals by nonisotopic chromosomal in situ suppression (CISS)-hybridization using a microdissection library as a probe. Hum Genet 87: 495-497.

Wells RA, Gemino GG, Krishna S, Buckle VJ, Reeders ST (1990) Telomere-related sequences at interstitial sites in the human genome. Genomics 8: 699-704.

Wienberg J, Jauch A, Stanyon R, Cremer T (1990) Molecular cytotaxonomy of primates by chromosomal in situ suppression hybridization. Genomics 8: 347-350.

Wienberg J, Stanyon R, Jauch A, Cremer T (1992) Homologies in human and Maccaca fuscata chromosomes revealed by in situ suppression hybridization with human chromosome specific DNA libraries. Chromosoma 101: 265-270.

Yunis JJ, Prakash O 1982 The origin of man: a chromosomal pictorial legacy. Science 215: 1525-1529. 